\title{
Wrapped Skew Laplace Distribution on Integers: A New Probability Model for Circular Data
}

\author{
K. Jayakumar ${ }^{1}$, Sophy Jacob ${ }^{2}$ \\ ${ }^{1}$ University of Calicut, Kerala, India \\ ${ }^{2}$ MES Asmabi College, Kerala, India \\ Email: \{jkumar19, sophyjacob\}@rediffmail.com
}

Received September 19, 2011; revised October 21, 2011 ; accepted October 31, 2011

\begin{abstract}
In this paper we propose a new family of circular distributions, obtained by wrapping discrete skew Laplace distribution on $\mathrm{Z}=0, \pm 1, \pm 2$, around a unit circle. In contrast with many wrapped distributions, here closed form expressions exist for the probability density function, the distribution function and the characteristic function. The properties of this new family of distribution are studied.
\end{abstract}

Keywords: Circular Distributions; Trigonometric Moments; Wrapped Laplace Distribution

\section{Introduction}

Circular data arise in various ways. Two of the most common correspond to circular measuring instruments, the compass and the clock. Data measured by compass usually include wind directions, the direction and orientations of birds and animals, ocean current directions, and orientation of geological phenomena like rock cores and fractures. Data measured by clock includes times of arrival of patients at a hospital emergency room, incidences of a disease throughout the year, where the calendar is regarded as a one-year clock. Circular or directional data also arise in many scientific fields, such as Biology, Geology, Meteorology, Physics, Psychology, Medicine and Astronomy [1].

Study on directional data can be dated back to the 18th century. In 1734 Daniel Bernoulli proposed to use the resultant length of normal vectors to test for uniformity of unit vectors on the sphere [2]. In 1918 von Mises introduced a distribution on the circle by using characterization analogous to the Gauss characterization of the normal distribution on a line [2]. Later, interest was renewed in spherical and circular data by [3-5].

Circular distributions play an important role in modeling directional data which arise in various fields. In recent years, several new unimodal circular distributions capable of modeling symmetry as well as asymmetry have been proposed. These include, the wrapped versions of skew normal [6], exponential [7] and Laplace [8].

Wrapped distributions provide a rich and useful class of models for circular data.

The special cases of the wrapped normal, wrapped Pois- son, wrapped Cauchy are discussed in [9]. We give a brief description of circular distribution in Section 2. In Section 3 we introduce and study Wrapped Discrete Skew Laplace Distribution. Section 4 deals with the estimation of the parameters using the method of moments.

\section{Circular Distributions}

A circular distribution is a probability distribution whose total probability is concentrated on the circumference of a circle of unit radius. Since each point on the circumference represents a direction, it is a way of assigning probabilities to different directions or defining a directional distribution. The range of a circular random variable $\Theta$ measured in radians, may be taken to be $(0,2 \pi]$ or $[-\pi, \pi]$.

Circular distributions are of two types: they may be discrete - assigning probability masses only to a countable number of directions, or may be absolutely continuous. In the latter case, the probability density function $f(\theta)$ exists and has the following basic properties.

1) $f(\theta) \geq 0$

2) $\int_{0}^{2 \pi} f(\theta) \mathrm{d} \theta=1$

3) $f(\theta)=f(\theta+2 \pi k)$, for any integer $k$. That is $f(\theta)$ is periodic with period $2 \pi$.

\section{Wrapped Distributions}

One of the common methods to analyze circular data is known as wrapping approach [10]. In this approach, given a known distribution on the real line, we wrap it 
around the circumference of a circle with unit radius. Technically this means that if $X$ is a random variable on the real line with distribution function $F(x)$, the random variable $X_{w}$ of the wrapped distribution is defined by

$$
X_{w}=X(\bmod 2 \pi)
$$

and the distribution function of $X_{w}$ is given by

$$
\begin{aligned}
& F_{w}(\theta)=\sum_{k=-\infty}^{\infty}[F(\theta+2 \pi k)-F(2 \pi k)], \\
& k=0, \pm 1, \pm 2, \cdots
\end{aligned}
$$

By this approach, we are accumulating probability over all the overlapping points $x=\theta, \theta \pm 2 \pi, \theta \pm 4 \pi, \cdots$ So if $g(\theta)$ represents a circular density and $f(x)$ the density of the real valued random variable, we have

$$
\begin{aligned}
& g(\theta)=\sum_{k=-\infty}^{\infty} f(\theta+2 \pi k), \\
& 0 \leq \theta \leq 2 \pi
\end{aligned}
$$

By this technique, both discrete and continuous wrapped distributions may be constructed. In particular, if $X$ has a distribution concentrated on the points $x=\frac{k}{2 \pi m}, k=0, \pm 1, \pm 2, \cdots$ and $m$ is an integer, the probability function of $X_{w}$ is given by

$$
\begin{aligned}
& \operatorname{Pr}\left(X_{w}=\frac{2 \pi r}{m}\right)=\sum_{k=-\infty}^{\infty} p(r+k m) \\
& r=0,1, \cdots, m-1
\end{aligned}
$$

where " $p$ " is the probability function of the random variable $X$.

\section{Wrapped Discrete Skew Laplace Distribution}

\subsection{Discrete Skew Laplace Distribution}

Discrete Laplace distribution was introduced by [11] following [12], who defined a discrete analogue of the normal distribution. The probability mass function of a general Discrete Normal random variable $Y$ can be written in the form

$$
\begin{aligned}
& P(Y=k)=\frac{f(k)}{\sum_{j=-\infty}^{\infty} f(j)}, \\
& k=0, \pm 1, \pm 2, \cdots
\end{aligned}
$$

where " $f$ " is the probability density function of a normal distribution with mean $\mu$ and variance $\sigma^{2}$ [13].

Using Equation (5), for any continuous random variable $X$ on $R$, we can define a discrete random variable $Y$ that has integer support on $Z$. When the skew Laplace density

$$
f(x)=\frac{1}{\sigma} \frac{\kappa}{1+\kappa^{2}}\left\{\begin{array}{l}
\mathrm{e}^{\frac{-|x|}{\kappa \sigma}}, \& x<0 \\
\mathrm{e}^{-\frac{\kappa|x|}{\sigma}}, \& x \geq 0
\end{array}\right.
$$

where, $\kappa>0$, are inserted into Equation (5), the probability mass function of the resulting discrete distribution takes an explicit form in terms of the parameters $p^{*}=$ $\mathrm{e}^{-\frac{\kappa}{\sigma}}$ and $q^{*}=\mathrm{e}^{-\frac{1}{\kappa \sigma}}$.

Definition 3.1 $A$ random variable $Y$ has a discrete skew Laplace distribution with parameters $p^{*} \in(0,1)$ and $q^{*} \in(0,1)$ denoted by DSL $\left(p^{*}, q^{*}\right)$, if

$$
\begin{aligned}
f(k) & =P[Y=k] \\
& =\frac{\left(1-p^{*}\right)\left(1-q^{*}\right)}{1-p^{*} q^{*}}\left\{\begin{array}{l}
p^{* *}, k=0,1,2, \cdots \\
q^{* k \mid}, k=0,-1,-2, \cdots
\end{array}\right.
\end{aligned}
$$

The characteristic function of $Y$ is given by

$$
\Phi(t)=\frac{\left(1-p^{*}\right)\left(1-q^{*}\right)}{\left(1-p^{*} e^{i t}\right)\left(1-q^{*} e^{i t}\right)}, t \in R
$$

In this paper, we study the probability distribution obtained by wrapping discrete skew Laplace distribution on $Z=0, \pm 1, \pm 2, \cdots$ around a unit circle.

As we know, reduction modulo $2 \pi$ wraps the line onto the circle, reduction modulo $2 \pi m$ (if $m$ is a positive integer) wraps the integers onto the group of $m^{\text {th }}$ root of 1 , regarded as a subgroup of the circle. That is, if $X$ is a random variable on the integers, then $\Theta$, defined by

$$
\Theta=2 \pi X(\bmod 2 \pi m)
$$

is a random variable on the lattice $\frac{2 \pi r}{m}, r=0,1, \cdots, m-1$ on the circle. The probability function of $\Theta$ is given by Equation (4).

In particular, if $X$ has a discrete skew Laplace distribution with parameters $p^{*}$ and $q^{*}$, then the probability distribution of the wrapped random variable $\Theta=\frac{2 \pi r}{m}$ is given by

$$
\begin{aligned}
P\left(\Theta=\frac{2 \pi r}{m}\right)= & \sum_{k=-\infty}^{\infty} p(r+k m), r=0,1, \cdots, m-1 \\
= & \sum_{k=-\infty}^{\infty} \frac{(1-p)(1-q) q^{|r+k m|}}{1-p q} \\
& +\frac{(1-p)(1-q)}{1-p q} p^{r} \\
& +\sum_{k=-\infty}^{\infty} \frac{(1-p)(1-q) p^{r+k m}}{1-p q}
\end{aligned}
$$

where $p=p^{*}(\bmod 2 \pi m)$ and 


$$
\begin{aligned}
q=q^{*}(\bmod 2 \pi m) & \\
= & \frac{(1-p)(1-q)}{1-p q}\left[\sum_{k=-\infty}^{-1} q^{-(r+k m)}+p^{r}+\sum_{k=1}^{\infty} p^{(r+k m)}\right] \\
=\frac{(1-p)(1-q)}{1-p q} & {\left[\left(1-p^{m}\right)+p^{r}\left(1-p^{m}\right)\left(1-q^{m}\right)\right.} \\
& \left.+p^{m+r}\left(1-q^{m}\right)\right], r=0,1, \cdots, m-1 \\
= & \frac{(1-p)(1-q)}{1-p q}\left[\frac{q^{m-r}\left(1-p^{m}\right)+p^{r}\left(1-q^{m}\right)}{\left(1-p^{m}\right)\left(1-q^{m}\right)}\right]
\end{aligned}
$$

for $r=0,1, \cdots, m-1$ and $p, q(0,1)$

Again, we have

$$
\sum_{r=0}^{m-1} p_{w}(\theta)=1
$$

Hence $P_{w}(\cdot)$ represents a probability distribution.

Definition 3.2 An angular random variable " $\Theta$ " is said to follow wrapped skew Laplace distribution on integers with parameters $p, q$ and $m$ if its probability mass function is given by

$$
p_{w}(\theta)=\frac{(1-p)(1-q)}{1-p q}\left[\frac{q^{m-r}\left(1-p^{m}\right)+p^{r}\left(1-q^{m}\right)}{\left(1-p^{m}\right)\left(1-q^{m}\right)}\right]
$$

$r=0,1, \cdots, m-1$ and $p, q \in(0,1)$ and we denote it by

$\operatorname{WDSL}(p, q, m)$.

Following are the graphs of wrapped discrete skew Laplace distribution for various values of $\kappa, \sigma$ and $\mathrm{m}$. In Figure 1, the graph of the pdf of wrapped discrete skew Laplace distribution for $\kappa=0.25, \sigma=1$ and for $m=5,10$, 20,30, 40, 50 and 100 are given.

In Figure 2, the graph of the pdf of wrapped discrete skew Laplace distribution for $\kappa=0.5, \sigma=1$ and for $m=5$, $10,20,30,40,50$ and 100 are given. The graph of the pdf of wrapped discrete skew Laplace distribution for $\kappa=$ $0.25, \sigma=1$ and for $m=5,10,20,30,40,50$ and 100 are given in Figure 3.

\subsection{Special Cases}

When either " $p$ " or " $q$ " converges to zero, we obtain the following two special cases: $\Theta \sim \operatorname{WDSL}(p, 0, m)$ with $p \in(0,1)$ is a wrapped geometric distribution with probability mass function

$$
P\left(\Theta=\frac{2 \pi r}{m}\right)=\frac{(1-p) p^{r}}{1-p^{m}}, r=0,1, \cdots, m-1 .
$$

while $\Theta \sim \operatorname{WDSL}(0, q, m)$ with $q \in(0,1)$ is a wrapped geometric distribution with probability mass function

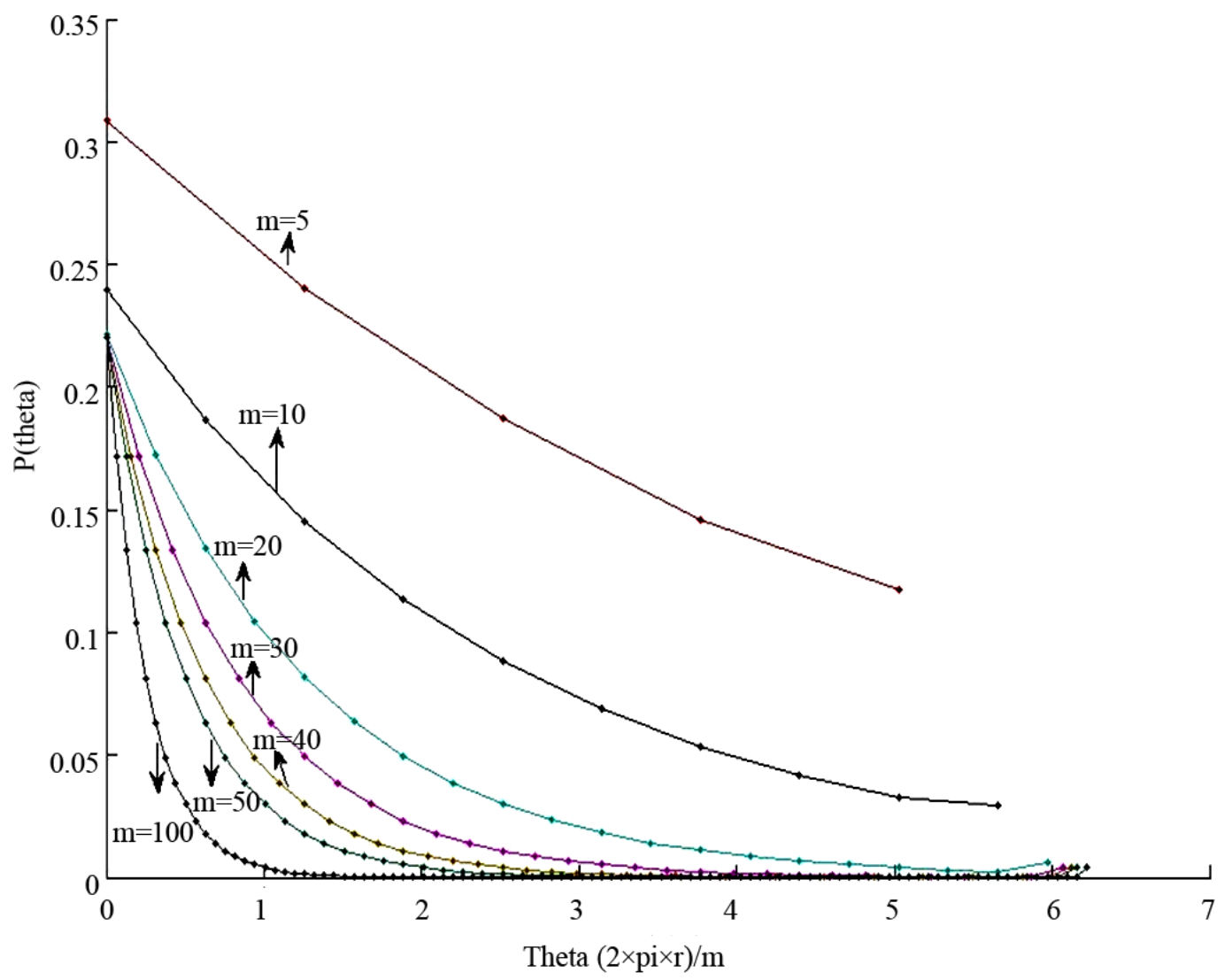

Figure 1. Wrapped discrete skewed Laplace distribution for $\sigma=1, \kappa=0.25$ and for different values of " $m$ ". 


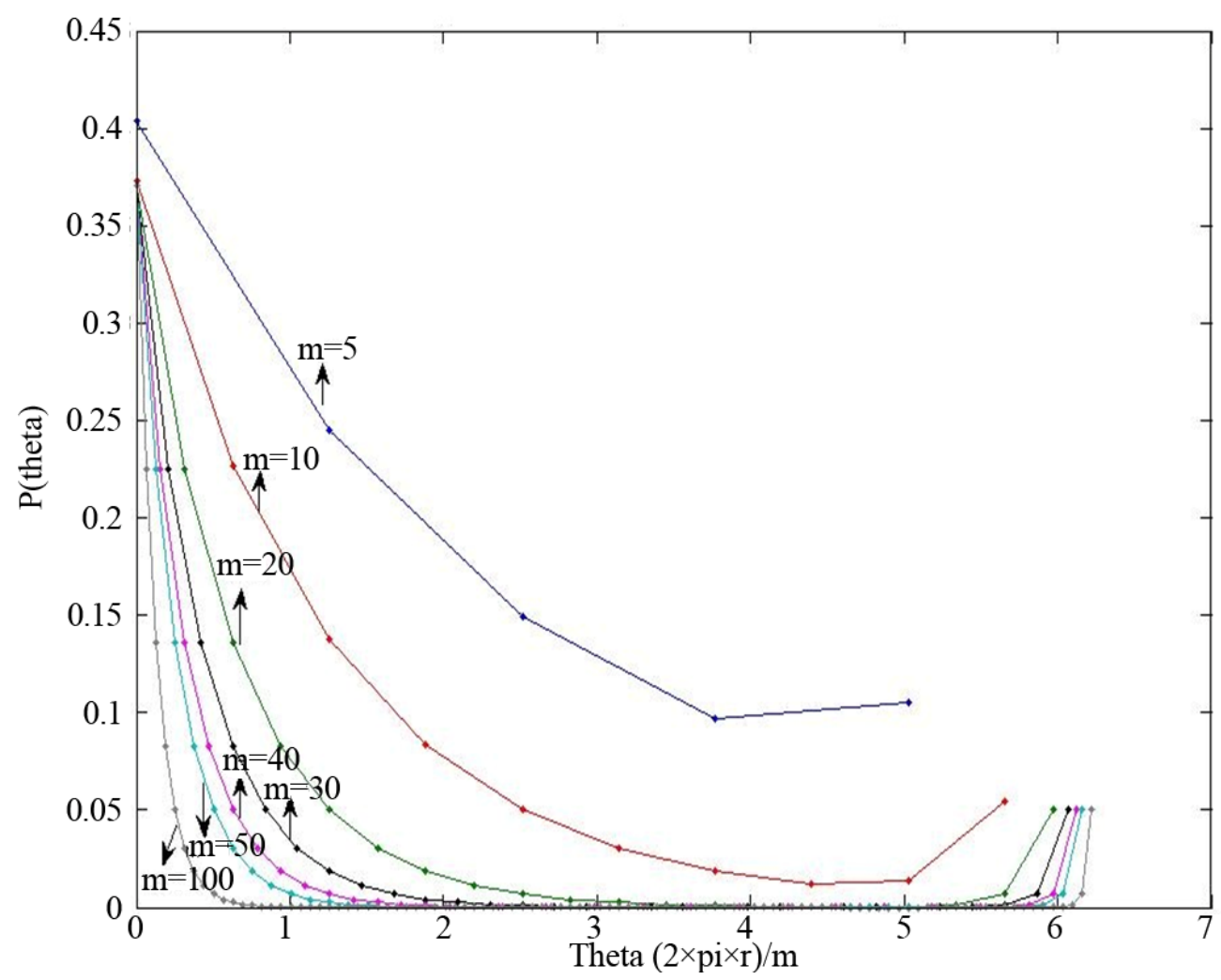

Figure 2. Wrapped discrete skewed Laplace distribution for $\sigma=1, \kappa=0.25$ and for different values of " $m$ ".

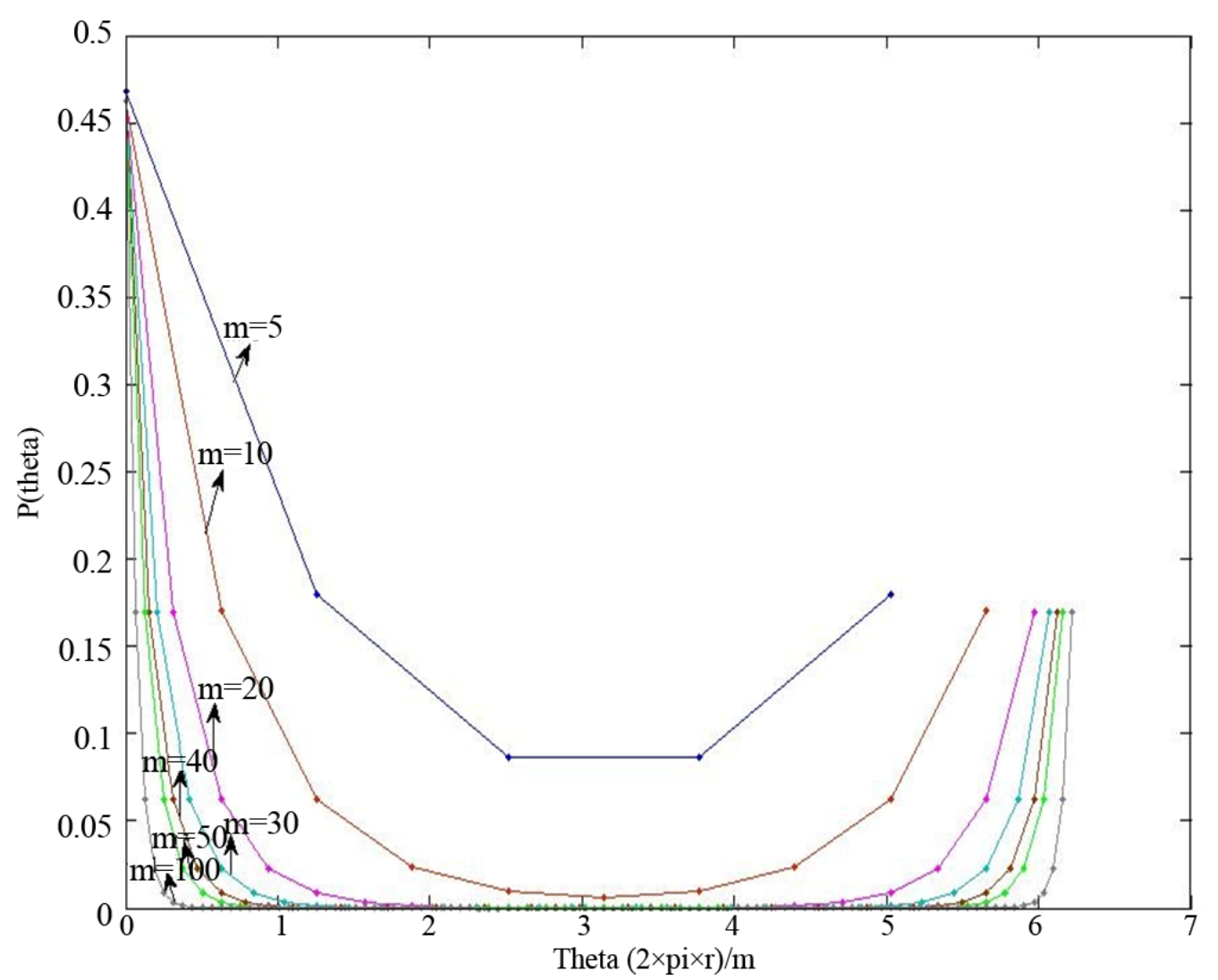

Figure 3. Wrapped discrete skewed Laplace distribution for $\sigma=1, \kappa=0.25$ and for different values of " $m$ ". 


$$
P\left(\Theta=\frac{2 \pi r}{m}\right)=\frac{(1-q) q^{-r}}{1-q^{m}}, r=0,-1, \cdots,-(m-1) .
$$

when $p=q$, we have

$$
\begin{aligned}
& P\left(\Theta=\frac{2 \pi r}{m}\right)=\frac{(1-p)\left(p^{m-r}+p^{r}\right)}{(1+p)\left(1-p^{m}\right)}, \\
& r=0,1, \cdots,(m-1)
\end{aligned}
$$

which is the probability mass function of wrapped discrete Laplace distribution.

\subsection{Distribution Function of $\operatorname{WDSL}(p, q, m)$}

The distribution function, $F(\theta)$ is given by

$$
\begin{gathered}
F(\theta)=\sum_{r=0}^{k} \frac{(1-p)(1-q)}{1-p q}\left[\frac{q^{m-r}\left(1-p^{m}\right)+p^{r}\left(1-q^{m}\right)}{\left(1-p^{m}\right)\left(1-q^{m}\right)}\right] \\
=\frac{q^{m-k}(1-p)\left(1-p^{m}\right)+(1-q)\left(1-q^{m}\right)\left(1-p^{k+1}\right)}{(1-p q)\left(1-p^{m}\right)\left(1-q^{m}\right)} \\
r=0,1, \cdots,(m-1) .
\end{gathered}
$$

\subsection{Probability Generating Function and Characteristic Function of $\operatorname{WDSL}(p, q, m)$}

The probability generating function of $\operatorname{WDSL}(p, q, m)$ is given by

$$
\begin{aligned}
P(s) & =\frac{(1-p)(1-q)}{(1-p q)\left(1-p^{m}\right)\left(1-q^{m}\right)} \sum_{r=0}^{m-1}\left[q^{m}\left(1-p^{m}\right)\left(\frac{s}{q}\right)^{r}+\left\{\left(1-p^{m}\right)\left(1-q^{m}\right)+p^{m}\left(1-q^{m}\right)\right\}(s p)^{r}\right] \\
& =\frac{(1-p)(1-q)}{(1-p q)\left(1-p^{m}\right)\left(1-q^{m}\right)}\left[\frac{\left(1-p^{m}\right)\left(q^{m}-s^{m}\right) q}{q-s}+\frac{\left(1-(s p)^{m}\right)\left(1-q^{m}\right)}{1-s p}\right]
\end{aligned}
$$

Also, we have $P(1)=1$. when $s=\mathrm{e}^{i 2 \pi n / m}$ we have

$$
\begin{gathered}
P\left(\mathrm{e}^{(i 2 \pi n) / m}\right)=\frac{(1-p)(1-q)}{\left(1-p \mathrm{e}^{(i 2 \pi n) / m}\right)\left(1-q \mathrm{e}^{(-i 2 \pi n) / m}\right)} \quad \begin{array}{l}
\text { wrapped random variable, } X_{w}, \text { is } \Phi(n), \text { for } \\
n=0, \pm 1, \pm 2, \cdots \quad \text { [2]. Thus for the wrapped discrete skew } \\
\text { Laplace distribution, we have }
\end{array} \\
\begin{aligned}
\Phi(n)=E\left[\mathrm{e}^{i n \Theta}\right], \quad \text { for } n=0, \pm 1, \pm 2, \cdots \\
=E\left[\mathrm{e}^{\frac{i n 2 \pi r}{m}}\right], \text { for } r=0,1, \ldots, m-1 \\
=\sum_{r=0}^{m-1} \frac{(1-p)(1-q)}{(1-p q)\left(1-p^{m}\right)\left(1-q^{m}\right)}\left[q^{m-r}\left(1-p^{m}\right)+p^{r}\left(1-p^{m}\right)\left(1-q^{m}\right)+p^{m+r}\left(1-q^{m}\right)\right] \mathrm{e}^{\frac{i n 2 \pi r}{m}}
\end{aligned}
\end{gathered}
$$

where $n \neq 0(\bmod m)$

If $\Phi(t)$ is the characteristic function of a linear random variable $X$, then the characteristic function of the

On simplification it reduces to

$$
\Phi(n)=\frac{(1-p)(1-q)}{\left(1-p \mathrm{e}^{(i 2 \pi n) / m}\right)\left(1-q \mathrm{e}^{-i 2 \pi n / m}\right)}, \quad n=0, \pm 1, \pm 2, \cdots, n \neq 0(\bmod m)
$$

Again, we have

$$
\Phi(n)=\frac{(1-p)(1-q)}{1-p \mathrm{e}^{\frac{i 2 \pi n}{m}}-q \mathrm{e}^{\frac{-i 2 \pi n}{m}}+p q}=\frac{(1-p)(1-q)\left[1+p q-(p+q) \cos \left(\frac{n 2 \pi}{m}\right)+i(p-q) \sin \left(\frac{n 2 \pi}{m}\right)\right]}{\left[1+p q-(p+q) \cos \left(\frac{n 2 \pi}{m}\right)\right]^{2}+\left[(p-q) \sin \left(\frac{n 2 \pi}{m}\right)\right]^{2}}=\alpha_{n}+i \beta_{n}
$$

where

$$
\alpha_{n}=\frac{(1-p)(1-q)\left[1+p q-(p+q) \cos \left(\frac{n 2 \pi}{m}\right)\right]}{\left[1+p q-(p+q) \cos \left(\frac{n 2 \pi}{m}\right)\right]^{2}+\left[(p-q) \sin \left(\frac{n 2 \pi}{m}\right)\right]^{2}}
$$

and

$$
\beta_{n}=\frac{(1-p)(1-q)(p-q) \sin \left(\frac{n 2 \pi}{m}\right)}{\left[1+p q-(p+q) \cos \left(\frac{n 2 \pi}{m}\right)\right]^{2}+\left[(p-q) \sin \left(\frac{n 2 \pi}{m}\right)\right]^{2}}
$$


Proposition 3.1 If $\Theta \sim \operatorname{WDSL}(p, q, m)$ then $\Theta \underline{\underline{\mathrm{d}}} \Theta_{1}-\Theta_{2}$ where $\Theta_{1}$ and $\Theta_{2}$ are two independently distributed wrapped geometric random variables with probability mass functions

$$
P\left(\Theta_{1}=\frac{2 \pi r}{m}\right)=\frac{p(1-p)^{r}}{1-(1-p)^{m}}, P\left(\Theta_{2}=\frac{2 \pi r}{m}\right)=\frac{q(1-q)^{r}}{1-(1-q)^{m}}
$$

\section{Proof.}

We have,

$$
\begin{array}{r}
\Phi_{\Theta}(n)=\frac{(1-p)(1-q)}{\left(1-p \mathrm{e}^{(i 2 \pi n) / m}\right)\left(1-q \mathrm{e}^{(-i 2 \pi n) / m}\right)} \\
n=0, \pm 1, \pm 2, \cdots, \mathrm{n} \neq 0(\bmod m)
\end{array}
$$

and

$$
\Phi_{\Theta_{1}}(n)=\frac{1-p}{1-p \mathrm{e}^{(i 2 \pi n) / m}}, \Phi_{\Theta_{2}}(n)=\frac{1-q}{1-q \mathrm{e}^{(i 2 \pi n) / m}}
$$

Therefore,

$$
\Phi_{\Theta_{1}-\Theta_{2}}(n)=\frac{(1-p)(1-q)}{\left(1-p \mathrm{e}^{(i 2 \pi n) / m}\right)\left(1-q \mathrm{e}^{(-i 2 \pi n) / m}\right)}=\Phi_{\Theta}(n)
$$

\subsection{Infinite Divisibility}

We know that the geometric distribution with probability mass function, $P(X=k)=(1-u)^{k} u, u=0,1, \cdots$ is infinitely divisible, so wrapped geometric distribution is infinitely divisible. Hence $\Theta \sim \operatorname{WDSL}(p, q, m)$ is infinitely divisible. By the well known factorisation properties of geometric law [14], $\Theta \sim W D S L(p, q, m)$ admits a representation involving wrapped negative binomial distribution, $\Theta \underline{\underline{\mathrm{d}}} \Theta_{n_{1}}+\Theta_{n_{2}}+\cdots+\Theta_{n_{k}}, k=1,2, \cdots$ where $\Theta_{n_{j}}$ $\stackrel{\mathrm{d}}{=} W_{n_{1}}-W_{n_{2}}$.

Consider

$$
\begin{aligned}
\Phi_{\Theta_{n_{j}}}(n) & =\frac{(1-p)}{\left(1-p \mathrm{e}^{(i 2 \pi n) / m}\right)} \frac{(1-q)}{\left(1-q \mathrm{e}^{(-i 2 \pi n) / m}\right)} \\
& =\frac{1}{\left(\frac{1}{1-p}-\frac{p \mathrm{e}^{(i 2 \pi n) / m}}{1-p}\right) \frac{1}{\left(\frac{1}{1-q}-\frac{q \mathrm{e}^{(-i 2 \pi n) / m}}{1-q}\right)}} \\
& =\frac{1}{\left(A-B \mathrm{e}^{(i 2 \pi n) / m}\right)} \frac{1}{\left(C-D \mathrm{e}^{(-i 2 \pi n) / m}\right)}
\end{aligned}
$$

where $A, B, C, D>0, A-B=C-D=1$ and $A=\frac{1}{1-p}$, $B=\frac{p}{1-p,}, C=\frac{1}{1-q}, D=\frac{q}{1-q}$

Therefore,

$$
\begin{aligned}
& \Phi_{\Theta_{n_{1}}+\Theta_{n_{2}}+\ldots+\Theta_{n_{k}}}(n) \\
= & \prod_{j=1}^{k}\left[\frac{1}{\left(A-B \mathrm{e}^{(i 2 \pi n) / m}\right)} \frac{1}{\left(C-D \mathrm{e}^{(-i 2 \pi n) / m}\right)}\right] \\
= & {\left[\left(A-B \mathrm{e}^{(i 2 \pi n) / m}\right)\left(C-D \mathrm{e}^{(-i 2 \pi n) / m}\right)\right]^{-k} }
\end{aligned}
$$

\subsection{Stability with Respect to Geometric Summation}

Proposition 3.2. Let $\Theta_{1}, \Theta_{2}, \cdots$ be identically and independently distributed WDSL $(p, q, m)$ angular random variables, and let $N_{u}$ be a geometric random variable with probability mass function $(1-u)^{k-1} u$, $k=1,2, \cdots$, independent of $\Theta_{j}^{\prime} s$. Then the angular random variable $\Theta \stackrel{\mathrm{d}}{=} \sum_{j=1}^{N_{u}} \Theta_{j}(\bmod 2 \pi)$ has the WDSL $(s, r, m)$ distribution with

$$
s=\frac{2 p}{\left\{p+q+(1-p)(1-q) u+\sqrt{[p+q+(1-p)(1-q) u]^{2}-4 p q}\right\}} \text { and } r=\frac{s q}{p}
$$

\section{Proof.}

Let $\Theta_{1}, \Theta_{2}, \cdots$ be identically and independently distributed angular random variables following $\operatorname{WDSL}(p, q, m)$ and $N_{u}$ is a geometric random variable with mean $1 / u$. Conditioning on the distribution of $N_{u}$ we can write the characteristic function of the right hand side of the above equation as (Equation (20))

$$
\begin{aligned}
& E\left[\mathrm{e}^{i\left(\Theta_{1}+\cdots+\Theta_{N_{u}}\right)(\bmod 2 \pi)}\right]=\sum_{k=1}^{\infty} E\left[\mathrm{e}^{i\left(\Theta_{1}+\cdots+\Theta_{k}\right)}\right](1-u)^{k-1} u \\
& =\frac{u \Phi(p, q, m)}{1-(1-u) \Phi(p, q, m)} \\
& =\frac{u(1-p)(1-q)}{\left(1-p \mathrm{e}^{i 2 \pi n / m}\right)\left(1-q \mathrm{e}^{(-i 2 \pi n) / m}\right)-(1-u)(1-p)(1-q)}
\end{aligned}
$$

Now we show that the above function coincides with the characteristic function of $\operatorname{WDSL}(s, r, m)$ distribution with $\mathrm{s}$ and $\mathrm{r}$ as given above. Setting Equation (20) equal to $\Phi(n / s, r)$, which is the characteristic function of $\operatorname{WDSL}(s, r, m)$ distribution, produces the equation

$$
\begin{aligned}
\Phi_{u, m}(n) & =\frac{(1-s)(1-r)}{\left(1-s \mathrm{e}^{\frac{i 2 \pi n}{m}}\right)\left(1-r \mathrm{e}^{\frac{-i 2 \pi n}{m}}\right)} \\
& =\frac{u(1-p)(1-q)}{\left(1-p \mathrm{e}^{\frac{i 2 \pi n}{m}}\right)\left(1-q \mathrm{e}^{\frac{-i 2 \pi n}{m}}\right)-(1-u)(1-p)(1-q)}
\end{aligned}
$$


That is,

$$
\begin{array}{r}
\left(1-s \mathrm{e}^{\frac{i 2 \pi n}{m}}\right)\left(1-r \mathrm{e}^{\frac{-i 2 \pi n}{m}}\right)(1-p)(1-q) u \\
=(1-s)(1-r)\left[\left(1-p \mathrm{e}^{\frac{i 2 \pi n}{m}}\right)\left(1-q \mathrm{e}^{\frac{-i 2 \pi n}{m}}\right)\right. \\
-(1-u)(1-p)(1-q)]
\end{array}
$$

which should hold for each $p, q$. This will happen when the following two equations hold simultaneously.

$$
\begin{aligned}
& (1-p)(1-q) u r=(1-s)(1-r) q \\
& (1-p)(1-q) u s=(1-s)(1-r) p
\end{aligned}
$$

Dividing Equation (23) by Equation (24), we get $r=\frac{s q}{p}$. Substituting the value of $\mathrm{r}$ in Equation (23) we get

$$
(1-p)(1-q)((u s q) / p)=(1-s)(1-(s q / p)) q
$$

On simplification, Equation (25) reduces to

$$
q s^{2}-s[q+p+(1-p)(1-q) u]+p=0
$$

That is, $f(s)=q s^{2}-s[q+p+(1-p)(1-q) u]+p$.

Since $p, q>0, f(0)=p>0$, and $f(1)=-u(1-p)(1-q)<0$.

Therefore, $f(\mathrm{~s})$ admits a unique solution in the interval $(0,1)$ and is given by

$$
\begin{aligned}
& =\frac{q+p+(1-p)(1-q) u \pm \sqrt{[q+p+(1-p)(1-q) u]^{2}-4 q p}}{2 q} \\
& =\frac{2 p}{q+p+(1-p)(1-q) u \pm \sqrt{[q+p+(1-p)(1-q) u]^{2}-4 q p}}
\end{aligned}
$$

Remark 3.1. Wrapped discrete skew Laplace distribution is infinitely divisible since a circular random variable obtained by wrapping an infinitely divisible random variable is infinitely divisible by $[1,11]$.

\subsection{Trigonometric Moments}

The $n^{\text {th }}$ trigonometric moment of the WDSL $(p, q, m)$ is given by

$$
\Phi_{\Theta}(n)=\frac{(1-p)(1-q)}{\left(1-p \mathrm{e}^{\frac{i 2 \pi n}{m}}\right)\left(1-q \mathrm{e}^{\frac{-i 2 \pi n}{m}}\right)}
$$

The above expression can also be expressed in the form

$$
\begin{aligned}
\Phi_{\Theta}(n) & =(1-p)(1-q)\left\{\left[1+p q-(p+q) \cos \left(\frac{n 2 \pi}{m}\right)\right]^{2}+\left[(p-q) \sin \left(\frac{n 2 \pi}{m}\right)\right]^{2}\right\}^{\frac{-1}{2}} \mathrm{e}^{i \tan ^{-1}}\left(\frac{(p-q) \sin \left(\frac{n 2 \pi}{m}\right)}{1+p q-(p+q)\left(\cos \left(\frac{n 2 \pi}{m}\right)\right)}\right) \\
& =\rho_{n} \mathrm{e}^{i \mu_{n}}
\end{aligned}
$$

where $\rho_{n} \in[0,1]$ is the $p$ th mean resultant length and and $\mu_{n} \in[0,2 \pi)$ is the $p$ th mean direction, for $n=1,2, \cdots$,

$$
\begin{aligned}
\rho_{n}=(1-p)(1-q)\{[1 & +p q-(p+q) \cos ((n 2 \pi) / m)]^{2} \\
& \left.+[(p-q) \sin ((n 2 \pi) / m)]^{2}\right\}^{\frac{-1}{2}}
\end{aligned}
$$

$$
\mu_{n}=\tan ^{-1}\left(\frac{(p-q) \sin \left(\frac{n 2 \pi}{m}\right)}{1+p q-(p+q)\left(\cos \left(\frac{n 2 \pi}{m}\right)\right)}\right)
$$

The length of the mean resultant vector,

$$
\rho=\rho_{1}=\sqrt{\alpha_{1}^{2}+\beta_{1}^{2}}=\frac{(1-p)(1-q)}{\sqrt{\left[1+p q-(p+q) \cos \left(\frac{n 2 \pi}{m}\right)\right]^{2}+\left[(p-q) \sin \left(\frac{n 2 \pi}{m}\right)\right]^{2}}}
$$

and the mean direction,

$$
\mu=\mu_{1}=\tan ^{-1}\left(\frac{\beta_{1}}{\alpha_{1}}\right)=\tan ^{-1}\left(\frac{(p-q) \sin \left(\frac{2 \pi}{m}\right)}{1+p q-(p+q) \cos \left(\frac{2 \pi}{m}\right)}\right)
$$


The circular variance,

$$
V_{0}=1-\rho=1-\frac{(1-p)(1-q)}{\sqrt{\left[1+p q-(p+q) \cos \left(\frac{n 2 \pi}{m}\right)\right]^{2}+\left[(p-q) \sin \left(\frac{n 2 \pi}{m}\right)\right]^{2}}}
$$

The circular standard deviation,

$$
\sigma_{0}=\sqrt{-2 \ln \rho}=\sqrt{\ln \left[\frac{\left.\left[1+p q-(p+q) \cos \left(\frac{n 2 \pi}{m}\right)\right]^{2}+\left[(p-q) \sin \left(\frac{n 2 \pi}{m}\right)\right]^{2}\right]}{[(1-p)(1-q)]^{2}}\right]}
$$

The measure of skewness,

$$
\gamma_{1}^{0}=\bar{\beta}_{2} / V_{0}^{3 / 2} \text { where } \bar{\beta}_{2}=E[\sin \sin 2(\Theta-\mu)]
$$

The measure of kurtosis,

$$
\gamma_{2}^{0}=\frac{\bar{\alpha}_{2}-\left(1-V_{0}\right)^{4}}{V_{0}^{2}} \text { where } \bar{\alpha}_{2}=E[\cos 2(\Theta-\mu)]
$$

\section{Estimation}

\section{Method of Moments}

Let $\theta_{1}, \cdots, \theta_{k}$ be a random sample of size $n$ taken from the $\operatorname{WDSL}(p, q, m)$ distribution with parameters $p, q$, and $m$. Then the $n^{\text {th }}$ sample trigonometric moment about the zero direction,

$$
m_{n}^{\prime}=a_{n}+i b_{n}
$$

where

$$
a_{n}=\frac{1}{k} \sum_{j=1}^{k} \cos \left(n \theta_{j}\right)
$$

and

$$
b_{n}=\frac{1}{k} \sum_{j=1}^{k} \sin \left(n \theta_{j}\right)
$$

Corresponding population moment is $\Phi(n)=\alpha_{n}+i \beta_{n}$ Equating the sample moments to the corresponding population moments, we get $\alpha_{n}=a_{n}$ and $\beta_{n}=b_{n}$ for $n=1,2, \cdots$. Thus, we have

$$
a_{1}=\frac{(1-p)(1-q)\left[1+p q-(p+q) \cos \left(\frac{2 \pi}{m}\right)\right]}{\left[1+p q-(p+q) \cos \left(\frac{2 \pi}{m}\right)\right]^{2}+\left[(p-q) \sin \left(\frac{2 \pi}{m}\right)\right]^{2}}
$$

and

$$
b_{1}=\frac{(1-p)(1-q)(p-q) \sin \left(\frac{2 \pi}{m}\right)}{\left[1+p q-(p+q) \cos \left(\frac{n 2 \pi}{m}\right)\right]^{2}+\left[(p-q) \sin \left(\frac{n 2 \pi}{m}\right)\right]^{2}}
$$

Using Equations (33)-(34) and for a fixed value of " $m$ " we can find estimates for " $p$ " and " $q$ ".

We have,

$$
\frac{a_{1}}{b_{1}}=\frac{1+p q-(p+q) \cos \left(\frac{2 \pi}{m}\right)}{(p-q) \sin \left(\frac{2 \pi}{m}\right)}
$$

That is

$$
a_{1}(p-q) \sin \left(\frac{2 \pi}{m}\right)=b_{1}\left[1+p q-(p+q) \cos \left(\frac{2 \pi}{m}\right)\right]
$$

which gives

$$
p=\frac{b_{1} q \cos \left(\frac{2 \pi}{m}\right)-a_{1} q \sin \left(\frac{2 \pi}{m}\right)-b_{1}}{b_{1} q-b_{1} \cos \left(\frac{2 \pi}{m}\right)-a_{1} \sin \left(\frac{2 \pi}{m}\right)}
$$

Substituting the value of " $p$ " in terms of " $q$ " in Equation (33) or in Equation (34) we will get an equation in " $q$ " and solving that we can find the estimate of " $q$ " and thus " $p$ ".

\section{REFERENCES}

[1] K. V. Mardia and P. E. Jupp, "Directional Statistics," 2nd Edition, Wiley, New York, 2001.

[2] K. V. Mardia, "Statistics of Directional Data," Academic Press, London, 1972.

[3] R. A. Fisher, "Dispersion on a Sphere," Proceedings of the Royal Society of London A, Vol. 217, No. 1130, 1953, pp. 295-305. doi:10.1098/rspa.1953.0064

[4] J. A. Greenwood and D. Durand, "The Distribution of Length and Components of the Sum of $\mathrm{n}$ Random Unit Vectors," Annals of Mathematical Statistics, Vol. 26, No. 2, 1955, pp. 233-246. doi:10.1214/aoms/1177728540

[5] G. S. Watson and E. J. William, "On the Construction of Significance Tests on the Circle and the Sphere," Biometrika, Vol. 43, 1956, pp. 344-352.

[6] A. Pewsey, "A Wrapped Skew-Normal Distribution on the Circle," Communications in Statistics: Theory and 
Methods, Vol. 29, No. 11, 2000, pp. 2459-2472. doi:10.1080/03610920008832616

[7] S. R. Jammalamadaka and T. J. Kozubowski, "A Wrapped Exponential Circular Model," Proceedings of the Andhra Pradesh Academy of Sciences, Vol. 5, 2001, pp. 43-56.

[8] S. R. Jammalamadaka and T. J. Kozubowski, “A New Family of Circular Models: The Wrapped Laplace Distributions," Advances and Applications in Statistics, Vol. 3, No. 1, 2003, pp. 77-103.

[9] S. R. Jammalamadaka and A. Sengupta, "Topics in Circular Statistics," World Scientific, Singapore, 2001. doi:10.1142/9789812779267

[10] P. E. Jupp and K. V. Mardia, “A Unified View of the Theory of Directional Statistics, 1975-1988," International Statistical Review, Vol. 57, No. 3, 1989, pp. 261-
294. doi: $10.2307 / 1403799$

[11] S. Inusah and T. J. Kozubowski, "A Discrete Analogue of the Laplace Distribution," Journal of Statistical Planning and Inference, Vol. 136, No. 3, 2006, pp. 1090-1102. doi:10.1016/j.jspi.2004.08.014

[12] A. W. Kemp, "Characterization of a Discrete Normal Distribution," Journal of Statistical Planning and Inference, Vol. 63, No. 2, 1997, pp. 223-229. doi:10.1016/S0378-3758(97)00020-7

[13] P. J. Szablowski, "Discrete Normal Distribution and Its Relationship with Jacobi Theta Functions," Statistics and Probability Letters, Vol. 52, No. 3, 2001, pp. 289-299. doi:10.1016/S0167-7152(00)00223-6

[14] W. Feller, "An Introduction to Probability Theory and Its Applications,” John Wiley \& Sons, New York, 1957. 